\title{
EDITORIAL
}

\section{A special section on Solar Cells, Light Emitting Diodes and Photodetectors}

\author{
Jiang TANG (ه) \\ Wuhan National Laboratory for Optoelectronics, Huazhong Univesity of Science and Technology, Wuhan 430074, China
}

(C) Higher Education Press and Springer-Verlag Berlin Heidelberg 2015

Solar cells, light emitting diodes and photodetectors are the three typical optoelectronic devices that play an important role in the welfare of human beings. Solar cell is a diode that absorbs solar irradiation and converts the incident photons into electron-hole pairs and then circulates and powers the external load. Light emitting diodes are also diodes that convert the injected electrons and holes, radiatively recombine within the active layer and then emit photons to light the world. Photodetector, normally either a diode or a resistor, also convert incident photons to output current, in analogy to solar cells. However, they distinct from solar cells in that photodetectors usually are biased during operation and their function is to detect light instead of utilize light for energy. All these three type of devices are actively and intensively studied in the academia, achieving better overall performance with lower cost being the ever-lasting goals. Consequently, new materials, new device configuration and new systematic integration are continuously proposed and investigated like bamboo shoots after a spring rain.

In this section, two review papers on the emerging photovoltaics, colloidal quantum dot (CQD) and copper zinc tin sulfoselenide (CZTSSe) solar cells, are presented. CQD photovoltaics utilize semiconductors in a nanoscale dimension as the active absorber layer, which possess the advantage of tunable band gap into the infrared and low cost solution processing. In the review paper presented by Prof. Xihua Wang from University of Alberta, the research progress in CQD photovoltaics from 2011, a best $5.1 \%$ efficiency, to 2015 , a record efficiency of $9.9 \%$, was thoroughly reviewed with special focus on the theoretical and experimental works that improve carrier collection efficiency and minimize recombination loss within CQD ensemble layer. CZTSSe is a new emerging absorber material for thin film photovoltaics in the hope that the expensive gradient indium in the well-established copper indium gallium diselenide (CIGS) solar cells could be replaced by inexpensive and earth abundant zinc and tin. Various strategies including vacuum based and solution based process were adopted to produce efficient CZTSSe solar cells. In the review paper written by Dr. Jie Zhong and Prof. Jiang Tang from Wuhan National Laboratory for Optoelectronics (WNLO), an environmentally friendly solution process using benign solvents to produce CZTSSe inks is reviewed. Key steps associated with this process including ink preparation and stabilization, film fabrication, device integration and performance are carefully analyzed and summarized. In addition to the review paper, a research paper employing poly(dimethyl diallylammonium chloride) (PDDA) layers to reduce the work function of ITO substrate is also presented by Prof. Yan Shen from WNLO, reporting increased short circuit current and enhanced device performance after their modification.

In the other two topics, light emitting diode and photodetector, two research paper are presented, each focusing on one topic. Organic light emitting diodes consume less power and have larger fields of view than conventional LCDs and have shown many competitive applications in the market. In the paper presented by Prof. Zujin Zhao from Hangzhou Normal University and Prof. Benzhong Tang from The Hong Kong University of Science \& Technology, aggregation induced emission from melted tetraphenylethene (TPE) and 1,2,4,5-tetraphenyl-1H-imidazole (TPI) units mixture are explored for blue fluorophores and then successfully integrated into light emitting diodes demonstrating electroluminescence at $467 \mathrm{~nm}(\mathrm{CIE}=0.17,0.22)$ with good efficiencies of $3.17 \mathrm{~cd} \cdot \mathrm{A}^{-1}$ and external

Received July 31, 2015

E-mail: jtang@mail.hust.edu.cn 
quantum efficiency of $1.77 \%$. For photodetection, photoconductive photodetectors enjoy the very high sensitivity originated from the intrinsic gain despite at a process of relatively low response speed. In this issue, Prof. Changqing Chen from WNLO explored the potential application of hydrothermally synthesized $\mathrm{Bi}_{2} \mathrm{~S}_{3}$ nanorods for visible light detection and found quite sensitive photoresponse in their devices.

Thanks the authors for their wonderful contribution to this issue. We hope that these papers could spur some research interest and scientific insight in the dynamic and exciting research field of optoelectronic devices.

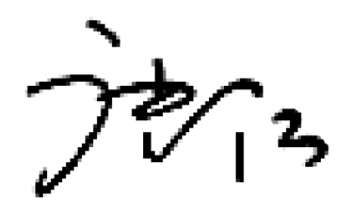

Jiang Tang

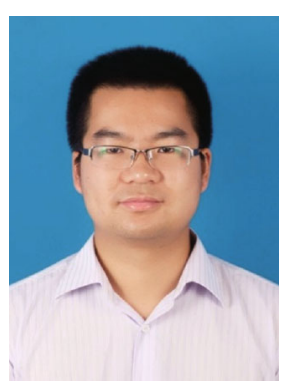

Jiang Tang is now a professor at Wuhan National Laboratory for Optoelectronics, Huazhong University of Science and Technology. He received his bachelor's degree from University of Science and Technology of China and his $\mathrm{Ph}$. D. degree from University of Toronto in the Department of Materials Science and Engineering under the supervision of Prof. Edward H. Sargent. His research interest is chalcogenide thin film solar cells and colloidal quantum dot optoelectronic devices. He is the research pioneer of antimony selenide $\left(\mathrm{Sb}_{2} \mathrm{Se}_{3}\right)$ thin film photovoltaics. 